\title{
The implications of breast cancer molecular phenotype for radiation oncology
}

\author{
Shirin Sioshansi, ${ }^{1,2}$, Kathryn E. Huber ${ }^{1}$ and David E. Wazer ${ }^{1,2 *}$ \\ 1 Department of Radiation Oncology, Tufts Medical Center, Tufts University School of Medicine, Boston, MA, USA \\ 2 Department of Radiation Oncology, Rhode Island Hospital, Warren Alpert School of Medicine at Brown University, Providence, RI, USA
}

\section{Edited by:}

Silvia Formenti, New York Langone

Cancer Institute, USA

\section{Reviewed by:}

Sean Collins, Georgetown University

Hospital, USA

Joshua Silverman, New York University

Medical Center, USA

Sylvia Adams, New York University, USA

\section{*Correspondence}

David E. Wazer, Department of Radiation Oncology, Tufts Medical Center, Box 359, Boston, MA 02111 USA.

e-mail:dwazer@tuftsmedicalcenter.org
The identification of distinct molecular subtypes of breast cancer has advanced the understanding and treatment of breast cancer by providing insight into prognosis, patterns of recurrence, and effectiveness of therapy. The prognostic significance of molecular phenotype with regard to distant recurrences and overall survival are well established in the literature and has been readily incorporated into systemic therapy management decisions. However, despite the accumulating data suggesting similar prognostic significance for locoregional recurrence, integration of molecular phenotype into local management decision making has lagged. Although there are some conflicting reports, collectively the literature supports a low risk of local recurrence $(L R)$ in the hormone receptor (HR) positive luminal subtypes compared to HR negative subtypes [triple negative (TN) and HER2-enriched]. The development of targeted therapies, such as trastuzumab for the treatment of HER2-enriched subtype, has been shown to mitigate the increased risk of LR. Unfortunately, no such remedy exists to address the increased risk of LR for patients with TN tumors, making it a clinical challenge for radiation oncologists. In this review we discuss the correlation between molecular subtype and LR following either breast conservation therapy or mastectomy. We also explore the possible mechanisms for increased LR in TN breast cancer and radiotherapeutic implications for this population, such as the safety of breast conservation, consideration of dose escalation, and the appropriateness of accelerated partial breast irradiation.

Keywords: breast, cancer, subtype, local, recurrence, triple, negative, radiotherapy

\section{INTRODUCTION}

Accurately estimating risk of local recurrence (LR) is essential to the practice of radiation oncology. In breast cancer, clinical and histopathologic risk factors for LR have been extensively studied and well-established. They include: margin status, young age, lymph node status, tumor size, lymphovascular invasion (LVI), and histologic grade (Clarke and Martinez, 1992). Computerized nomograms that calculate the risk of LR and aid local management decision making exist (IBTR!, 2011). However, our ability to accurately estimate LR risk remains limited reflecting the heterogeneity of the disease. Over the past decade, molecular profiling has improved our understanding of how each tumor is unique and should be treated accordingly. Individualized care, based on a tumor's genetic fingerprint (or surrogate subtype based on phenotypic markers) may 1 day govern adjuvant treatment decision making by identifying patients who are candidates for observation after breast conserving surgery, hypofractionation, accelerated partial breast irradiation (APBI), or dose escalation.

\section{MOLECULAR PHENOTYPING IN BREAST CANCER}

The first molecular distinction made in breast cancer was from the work in the 1960s and 1970s that identified and characterized estrogen receptor (ER) and progesterone receptor (PR) expression on breast cancer cells. This discovery quickly led to the development of antiestrogen therapies. Responsiveness of the tumor to hormonal therapy correlated directly with receptor expression and identified an important biological subdivision within breast cancer: those that were ER-positive and respond to antiestrogens and those that are ER-negative and are refractory to hormonal manipulation (Fisher et al., 1983). Another biologically distinct subset of breast cancer was discovered by novel work investigating the amplification of the human epidermal growth factor receptor 2 (HER2/ neu) oncogene in breast cancer (Slamon et al., 1987). The analysis of 189 primary breast tumors showed that the amplification of the HER2/neu oncogene occurred in $25-30 \%$ of the specimens and that this genomic alteration predicted poor clinical outcome, even after adjustment for other prognostic variables. Similar to the earlier breakthrough investigations of hormone receptors (HR) in breast cancer, this finding led to the development of targeted therapy and has had a significant impact on the outcome of women with HER2 overexpressing tumors (Piccart-Gebhart et al., 2005; Romond et al., 2005).

Gene expression profiling, which examines the relative expression of thousands of genes in a single sample simultaneously, led to the discovery of distinct molecular subtypes within breast cancer (Perou et al., 2000). These molecular subtypes have phenotypic diversity with regard to multiple clinical outcomes, including response to chemotherapy, disease-free survival, and overall survival. Multiple datasets have confirmed these molecular breast cancer subtypes which include at least two luminal subtypes (luminal A and B) that comprise most ER-positive breast cancer and are characterized by a high expression of HR-related genes; the basal-like subtype, which is characterized by a high expression of a unique "basal" signature that includes genes common to the breast 
myoepithelium, high expression of proliferation genes, and low expression of the ER signature and HER2 signatures; and the HER2enriched subtype, which is typified by high expression of HER2related and proliferation genes and low expression of HR-related genes. A fifth subtype, the normal breast subtype, has expression patterns similar to nonmalignant tissue and likely reflects sampling artifact (Sorlie et al., 2001, 2003; Sotiriou et al., 2003).

Molecular subtype has been shown to be significantly associated with differences in overall and relapse-free survival (Sorlie et al., 2001). The basal-like and HER2-enriched subtypes showed the poorest prognosis with shorter time to progression and lower overall survival. Patients belonging to the luminal A subtype had a considerably better prognosis compared with all groups, and those with the luminal B subtype had an intermediate outcome. The luminal subtypes are the most heterogeneous with regard to biology and outcomes (Perou et al., 2000; Sorlie et al., 2001, 2003; Sotiriou et al., 2003). Luminal A tumors have variable proliferation gene expression and also have highly variable prognostic signatures (Fan et al., 2006). Luminal B tumors, although still expressing the HR-related gene signature, do so at a lower level, have variable expression of the HER2 signature, and are generally more proliferative than the luminal A subtype. In multiple datasets, patients with luminal B tumors have worse outcome compared luminal A tumors, despite both usually being ER-positive.

Because of the technical limitations of performing microarray expression analysis on formalin fixed, paraffin-embedded tissue, the use of ER, PR, HER2, and cytokeratin (CK) immunohistochemical (IHC) markers has been used as a surrogate to molecular subtypes. The marker combinations that best matched the molecular profiles segregated the tumors into four groups (Table 1): (1) ER+ and/or PR+, HER2- for luminal A subtype; (2) ER+ and /or $\mathrm{PR}+, \mathrm{HER} 2+$ for luminal B subtype (although this is known to misclassify a significant portion); (3) ER-, PR-, HER2-, CK 5/6+, and/or epidermal growth factor receptor (EGFR)+ for basal-like subtype; and (4) ER-, PR-, and HER2+ for the HER2-enriched subtype (Nielsen et al., 2004; Carey et al., 2006). The limitation of this simplification is that the prognostic power of the subtypes is based on a complex gene expression signature and that these molecular profiles are only associated with these IHC markers and not synonymous. In fact, the molecular profiles have been found to have a more robust predictive value compared with the surrogate markers (Sorlie, 2004). However, the proxy use of the IHC markers has allowed for the analysis of large datasets and the discovery of important aspects of the biology of these tumor subtypes. In the future, it is possible that RT-PCR-based approaches, such as the

Table 1 | Immunohistochemically defined breast cancer molecular subtypes.

\begin{tabular}{llll}
\hline & ER & PR & HER2 \\
\hline Luminal A & ER (+) and/or PR (+) & & $(-)$ \\
Luminal B & ER (+) and/or PR (+) & & $(+)$ \\
HER2-enriched & $(-)$ & $(-)$ & $(+)$ \\
Triple negative & $(-)$ & $(-)$ & $(-)$ \\
\hline
\end{tabular}

$E R$, estrogen receptor; $P R$, progesterone receptor.
PAM50 assay, which can measure a set of 50 intrinsic genes from formalin fixed, paraffin-embedded tissue, will permit additional molecular subtyping in archival specimens (Parker et al., 2009).

A large population-based study using the IHC surrogate markers for molecular subtype examined the association of molecular subtype with clinical characteristics and found that the luminal A, luminal B, basal-like, and HER2-enriched subtypes differed significantly by age, race, menopausal status, lymph node involvement, histology group, tumor grade, and mitotic index (Carey et al., 2006). The most striking distribution was the overrepresentation of basal-like tumors in premenopausal black women (39\% in premenopausal black women vs. $14 \%$ in postmenopausal black women and $16 \%$ in non-black women of all ages, $p<0.001$ ). Subsequent studies have confirmed that basal-like tumors are more frequent in younger patients and black women (Bauer et al., 2007; Millikan et al., 2008). In addition patients with basal-like tumors tended to have aggressive features including high nuclear grade, high mitotic index, and unfavorable histology (meta-plastic, anaplastic, or undifferentiated high grade carcinomas). Paradoxically, this subtype was not associated with higher regional lymph node involvement. More recent analyses have provided supporting evidence for the decreased prevalence of lymph node metastasis in basal-like tumors and have shown a disconnect between tumors size and positive lymph nodes in this subtype (Crabb et al., 2008; Foulkes et al., 2009; Billar et al., 2010). This finding suggests a difference in mechanism of metastasis between luminal and basal tumors and highlights that these breast cancer molecular subtypes are distinct biological entities.

In addition to molecular subtypes developed through unsupervised hierarchical clustering algorithms, several other tailored molecular assays have been developed to specifically guide the use of adjuvant systemic therapy including the 21-gene recurrence score assay (Oncotype DX, Genomic Health, Redwood City, CA, USA; REF \#43 PAIK-NEED TO REORDER), the 70-gene recurrence score assay (MammaPrint, Agendia, Amsterdam, The Netherlands; van't Veer et al., 2002), and several others. These assays will likely further supplement molecular phenotype and our appreciation of the heterogeneous behavior of breast cancer.

\section{MOLECULAR PHENOTYPE AND LOCAL RECURRENCE}

The prognostic significance of molecular phenotype with regard to distant recurrences and overall survival are well established in the literature and has been readily incorporated into systemic therapy management decisions. However, despite the accumulating data suggesting similar prognostic significance for LR, integration of molecular phenotype into local management decision making has lagged. This may reflect some uncertainty regarding the correlation between LR risk and molecular phenotype as some of the earlier reports did not show a difference in LR by molecular phenotype (Haffty et al., 2006; Dent et al., 2007).

The first of these reports was a retrospective analysis of 482 women treated with breast conservation therapy (BCT) at Yale between 1980 and 2003 for which ER, PR, and HER2 data were available (Haffty et al., 2006). With a median follow-up of 7.9 years, they reported $17 \%$ LR rates in the triple negative (TN) and non-TN cohorts. However, they also reported a difference in nodal relapsefree survival of $94 \%$ in the TN cohort and $99 \%$ in the non-TN 
cohort $(p=0.05)$. This study reports relatively high rates of LR, which likely reflects the less aggressive systemic therapy typically used in patients in the early portion of the study period. In addition, this higher recurrence rate could also reflect a bias during the earlier portion of the study period to obtain IHC markers more often in women with higher risk tumors. This selection bias also explains the relatively high rate of TN tumors included in the study (25\% of the cohort). Another limitation of this study is that HER2 IHC stain was not confirmed with FISH, which is known to contribute to misclassification. Finally, the TNs were compared to all non-TNs as opposed to each molecular phenotype compared individually. The non-TN cohort LR rate was elevated by the inclusion of the HER2-enriched subtype that has been consistently shown to have a higher rate of LR in the pre-trastuzumab era (Nguyen et al., 2008; Millar et al., 2009; Voduc et al., 2010).

The second report that did not show a difference in LR by molecular phenotype was reported by Dent et al. (2007). This report was a database review of 1,601 women with invasive breast cancer with known ER, PR, and HER2 status treated between 1987 and 1997 at the Henrietta Banting Breast Cancer Centre. The majority of the subjects included in this analysis had palpable tumors and nearly half of the patients had positive lymph nodes. The analysis also included patients treated with mastectomy. With a median follow-up of 8.1 years, they reported no statistically significant difference in LR between the TN and non-TN (13 vs. 12\% $p=0.77$ ). Although they did not see a higher rate of LR, they did observe a shorter time to LR among the TN phenotype (2.8 vs. 4.2 years, $p=0.02$ ). Similar to Haffty et al. (2006) this report was subject to misclassification as FISH analysis was not available to confirm HER2 amplification. They also had a relatively high overall LR rate which reflects the earlier time period as well as the more advanced stage of tumors included. The TN cohort also had bigger tumors (63.5\% TN $>2 \mathrm{~cm}$ vs. $37.4 \%$ of non-TN, $p<0.0001)$ and higher rates of positive lymph nodes $(54.4 \%$ of TN vs. $45.6 \%$ of non$\mathrm{TN}, p=0.02$ ) and thus were potentially more likely to be treated with mastectomy and chemotherapy, which could confound the comparison of LR.

Another retrospective database review of 753 T1-2 breast cancers treated with BCT at Fox Chase Cancer Center also did not show a difference in isolated locoregional recurrence (LRR) at 5 years by molecular phenotype (Freedman et al., 2009). However, the total LRR [including LR with synchronous distant metastases (DM)] was higher among the TN subtype (5.3\%) compared to the HR positive cohort $(2.6 \%), p=0.05$. This report highlights the fact that DM is a major competing risk for the TN subtype and may artificially lower the LR rates in studies that routinely only document first site of recurrence. Similar to Dent et al. (2007), the authors also reported a shorter time to LR among the TN subtype.

In contrast to these reports, there is mounting evidence that molecular phenotype is prognostic for LR risk. Nguyen et al. (2008) published a retrospective review of 793 consecutive patients with invasive breast cancer treated with BCT from 1998 to 2001. With a median follow-up of 70 months, they reported 5-year LR rate of $0.8 \%$ for luminal A, $1.5 \%$ for luminal B, $8.4 \%$ for HER2-enriched, and $7.1 \%$ for TN. On multivariate analysis, HER2-enriched [adjusted hazard ratio $(\mathrm{AHR})=9.2, p=0.012$ ] and $\mathrm{TN}(\mathrm{AHR}=7.1$, $p=0.009)$ subtypes were associated with increased LR.
Millar et al. (2009) corroborated these results with a prospective database review of 498 patients treated with BCT. With 84 months of follow-up, they reported LRR rates of 5.1\% for luminal A, 8.7\% for luminal B, 17.3\% for basal, 15.4\% for HER2-enriched, and $12.5 \%$ for unclassified ( $p=0.012$ ). However, the rates of ipsilateral breast tumor recurrence (IBTR) were not statistically different by subtype. This study was unique because it used EGFR and CK 5/6 status in addition to the standard ER, PR, and HER2 receptors to approximate the basal-like molecular subtype with a specificity of $100 \%$ and a sensitivity of $76 \%$ (Cheang et al., 2008). This distinction separated the TN subtype into basal (EGFR+ and/or CK5/6+) and unclassified (negative for both EGFR and CK5/6).

Voduc et al. (2010) used a similar biomarker panel with the addition of Ki-67 and reported similar results with a series of 2,985 women treated between 1986 and 1992 in British Columbia. With median follow-up of 12 years, they showed the greatest difference by molecular subtype in LR ( $8 \%$ luminal A, 21\% HER2-enriched and $14 \%$ basal-like) and regional recurrence (RR; 3\% luminal A, 16\% HER2-enriched and $14 \%$ basal-like). This pattern was also maintained among the patients treated with mastectomy. Importantly, patients with positive surgical margins were excluded from the analysis, which likely resulted in reduced overall LR rates compared to other reports. A notable finding of this study was that the LRR rate of the TN-unclassified subtype was similar to that of the luminal subtypes and thus, the EGFR and CK5/6 markers appear to aid in determining which TNs are higher risk of LRR. TN tumors with high Ki-67 were also found to have an increased rate of LRR.

Billar et al. (2010) reported a retrospective review of 1,061 cases treated at the Mayo Clinic in Arizona between 2000 and 2008. With only 31 months of median follow-up, they showed a difference in LRR between the molecular phenotypes: $1 \%$ for the luminal A, 2.9\% for the HER2-positive tumors (luminal B and HER2-enriched), and $5.7 \%$ for the TNs $(p=0.001)$.

The difference in LR among the molecular subtypes also extends to the postmastectomy and postmastectomy radiation (PMRT) setting. A reanalysis of the Danish Breast Cancer Group (DBCG) PMRT (trials $82 \mathrm{~b}$ and $82 \mathrm{c}$ ) data by molecular phenotype showed increased LRR among the HER2-enriched and TN phenotypes (Kyndi et al., 2008). For the patients treated with mastectomy and PMRT, 15 year LRR rates were 3\% for luminal A, 3\% for luminal B, 21\% for HER2-enriched, and 15\% for TN.

A point of contention around these reports is that due to their retrospective design, the authors could not control for confounding factors such as systemic therapy. As expected, the HR negative subtypes were more likely to receive chemotherapy (Nguyen et al., 2008). An effort to address this issue was made by Albert et al. (2010) by limiting their analysis to tumors $<1 \mathrm{~cm}$ because such small tumors were less likely to be treated with chemotherapy (only $8 \%$ of their total cohort). Their series included 911 patients treated at MD Anderson Cancer Center between 1997 and 2002. With a median follow-up of 6 years, they showed statistically significant difference in LRR by receptor status for ER [ER(-) $10.6 \%$ vs. $\mathrm{ER}(+) 4.2 \%, p=0.016]$, PR [PR(-) 9\% vs. $\mathrm{PR}(+) 4.2 \%, p=0.009]$, and HER2 [HER2 $(+) 17.5 \%$ vs. HER2 $(-) 3.9 \%, p=0.009$ ]. The authors also analyzed LRR by combined HR and HER2 status. Eight year LRR rates were: 3.5\% for HR+HER2- (luminal A), $5.8 \%$ for HR-HER2- (TN), 13.4\% for HR+HER2+ (luminal B), 
and 29.2\% for the HR-HER2+ (HER2-enriched; $p=0.014)$. On multivariate analysis, ER/PR-negative and HER2-positive status independently predicted for LRR. The authors also ran multivariate analysis after excluding the 61 patients who received chemotherapy and ER/PR-negative status still maintained statistically significant correlation with LRR ( $p=0.005)$. Although this study is compelling, the need for prospective randomized trials to validate these findings remains.

\section{RADIOTHERAPEUTIC IMPLICATION OF MOLECULAR PHENOTYPE}

Although there are some conflicting reports, collectively the literature supports a low risk of LRR in the luminal phenotypes and increased risk of LRR in TN and HER2-enriched breast cancer phenotypes after BCT and mastectomy with PMRT (summarized in Table 2). This begs the question, what can be done to combat this risk? Trastuzumab, a monoclonal antibody to the HER2/ neu receptor, is the obvious answer for the HER2-enriched subtype as it has been shown to decrease LRR in HER2+ disease by approximately $50 \%$ in randomized trials (Piccart-Gebhart et al., 2005; Romond et al., 2005). None of the HER2-enriched patients received trastuzumab in the studies that demonstrated increased risk of LRR among this subtype. However, the report by Billar et al. (2010), the only study that included patients who were treated with trastuzumab, showed a low rate of LRR among the HER2+ patients $(2.9 \%)$. Thus, the addition of trastuzumab may eliminate or narrow the difference in LR rates for the HER2+ subtypes. Unfortunately, no such remedy exists to address the increased risk of LRR among the TN subtype, making it a clinical challenge for surgical and radiation oncologists.

Before postulating how we may be able to alter our practices to address the increased risk of LRR in TN breast cancer (TNBC), it is important to understand what may be the underlying potential mechanism of the increased risk of LRR. Existing theories include:

Table 2 | Summary of literature exploring the relationship of local recurrence rate and breast cancer molecular subtype.

\begin{tabular}{|c|c|c|c|c|}
\hline Author & $n$ & Median follow-up (years) & Local recurrence rate (\%) & $p$-value \\
\hline Nguyen et al. (2008) & 793 & 5.8 & $\begin{array}{l}\text { Luminal A: } 1.8 \\
\text { Luminal B: } 1.5 \\
\text { HER2: } 8.4 \\
\text { Basal/TN: } 7.1\end{array}$ & $\mathrm{SS}^{* *}$ \\
\hline Freedman et al. (2009) & 753 & $3.4-4$ & $\begin{array}{l}\text { Luminal: } 2.6^{+} \\
\mathrm{TN}: 5.3^{+}\end{array}$ & 0.05 \\
\hline Millar et al. (2009) & 498 & 7 & $\begin{array}{l}\text { Luminal A: } 5.1^{*} \\
\text { Luminal B: } 8.7^{*} \\
\text { HER2-enriched: } 15.4^{*} \\
\text { Basal-like: } 17.3^{*} \\
\text { Unclassified: } 12.5^{*}\end{array}$ & 0.012 \\
\hline Billar et al. (2010) & 1,061 & 2.6 & $\begin{array}{l}\text { Luminal A: } 1^{*} \\
\text { Luminal B and HER2-enriched: } 2.9^{*} \\
\text { TN: } 5.7^{*}\end{array}$ & 0.001 \\
\hline Albert et al. (2010) & 911 & 6 & $\begin{array}{l}\text { Luminal A: } 3.5^{*} \\
\text { Luminal B: } 13.4^{*} \\
\text { HER2-enriched: } 29.2^{*} \\
\text { TN: } 5.8^{*}\end{array}$ & 0.014 \\
\hline
\end{tabular}

*Isolated locoregional recurrence (LRR) rate, ${ }^{\dagger}$ total LRR (including LRR with synchronous distant metastasis), ${ }^{\ddagger} L R R$ after mastectomy and PMRT, ** stastistically significant (SS) differences between groups without p-value reported.

$T N$, triple negative; TNP, triple negative phenotype; SS, statistically significant; PMRT, postmastectomy radiation. 
(1) TNBC is radioresistant, (2) TNBC has a higher proliferative rate resulting in early failures, but with longer follow-up the luminal subtypes "catch up," (3) TNBC has an increased rate of residual disease after lumpectomy.

\section{1-IS TNBC RADIORESISTANT?}

The impact of molecular phenotype on the response to radiation was recently reported by a retrospective subset analysis of data from 1,000 patients enrolled in the Danish PMRT trials DBCG 82b and c (Kyndi et al., 2008). The patients had clinical features associated with an increased risk of LR following mastectomy including large primary tumors $(>5 \mathrm{~cm})$, invasion of skin or pectoralis fascia, or lymph node involvement. The premenopausal women (DBCG 82b) were treated with adjuvant cyclophosphamide, methotrexate, fluorouracil (CMF) chemotherapy while the postmenopausal women (DBCG 82c) received tamoxifen. All women were randomized to either receive or not receive PMRT. There was an overall survival benefit with PMRT in both the pre- and postmenopausal women (Overgaard et al., 1997, 1999). ER, PR, and HER2 status were not known at the time of the DBCG initial report and thus, Kyndi et al. (2008) retrospectively performed tissue microarray analysis for these receptors in order to analyze outcomes by molecular subtype. Of note, HER2 2+ IHC pattern on microarray was confirmed for gene amplification by FISH analysis. They found that the luminal A, luminal B, and TN subtypes had a statistically significant LRR benefit with PMRT, but only the luminal A subtype had a statistically significant overall survival benefit. The authors also noted that the TN subtype had a smaller proportional locoregional control benefit compared to the luminal A and luminal B subtype and concluded that increased radioresistance among the HR negative subtypes may explain, in part, the lack of improvement in survival.

However, this study did not have the statistical power to detect significant survival benefit from PMRT in the non-luminal A cohorts that had significantly fewer number of patients. In addition to lack of statistical power, another explanation for the lack of survival benefit among the TN subtype was the use of suboptimal systemic therapy. The women enrolled on these trials were at high risk for harboring micrometastases at time of diagnosis and thus systemic therapy is integral toward maximizing survival. The premenopausal women only received CMF chemotherapy which is now considered suboptimal systemic therapy for this high risk breast cancer population. The postmenopausal women received tamoxifen alone which is ineffective in TNBC. In addition, many studies have shown that TN subtype has a predilection for distant failure (Sorlie et al., 2001). As the risk of distant failure increases, locoregional control becomes less likely to translate into survival benefit. This theory is corroborated by a separate subgroup analysis by DBCG analyzing the same data based on good, intermediate, and high risk groups. They found that the largest translation of LRR benefit from PMRT to survival benefit was seen in the "good" prognosis group (Kyndi et al., 2009). This was despite the fact that the high risk group had a larger proportional LR benefit with PMRT. TNBC had a clear reduction of LRR with PMRT $(p=0.001)$ in these trials, but this improvement in local control very likely did not translate to a survival benefit because of the competing risk of distant failure in the setting of suboptimally treated advanced stage disease.
In Kyndi's study, the distant recurrence and overall survival rates among the TN phenotype was also comparable to the luminal phenotypes which is not consistent with other reports in the literature. This discrepancy could be a result of heterogeneity within TNs. For example, the Danish cohort of TNs likely represent fewer women of African descent and there is evidence that Caucasian (especially postmenopausal Caucasian) women may have better outcomes compared to women of African descent (Sachdev et al., 2010). Although this difference could represent differences in socioeconomic status and access to care, it could also be explained by differences in biology.

\section{2-IS THE POOR PROGNOSIS ASSOCIATED WITH TNBC TRANSIENT?}

The difference in LRR between the subtypes is most dramatic in the first few years of follow-up. Dent et al. (2007) showed that nearly all TN failures occur within the first 5 years, while the failure rate among the luminal subtypes continues with time. Thus, Dent et al. (2007) concluded that longer follow-up diminishes the difference in outcomes among the subtypes and that the poor prognosis of TNs is merely transient. However, longer follow-up does not eliminate the difference entirely as shown by Voduc et al. (2010) who reported statistically significant difference in LRR with a mature median follow-up of 12 years. The prolonged course of LR in luminal subtypes may reflect adjuvant hormonal therapy keeping LR dormant for many years and/or more indolent biology while the accelerated course of LR in TNBC may reflect the high proliferative genetic signature of the basal-like subtype (Sorlie et al., 2001). For this reason, accelerated hyperfractionated radiotherapy may be beneficial for the treatment of TNBC. This method of radiation therapy typically uses slightly smaller dose per fraction, but delivers more than 5 treatments per week. For example delivering 1.5 Gy per fraction 10 times per week with treatments delivered twice daily for five consecutive days. This would yield a weekly dose is $15 \mathrm{~Gy}$, which is $50 \%$ more dose than the weekly dose of $10 \mathrm{~Gy}$ given with standard fractionation of 2 Gy per fraction given for 5 consecutive days. Therefore, with accelerated hyperfractionation, the total radiation course could be completed over a shorter period of time, which minimizes the effect of ongoing tumor proliferation that occurs during treatment. This could improve local control in a tumor subtype with very rapid proliferation such as the TNBC, and should be considered as part of a prospective clinical trial for women with this molecular subtype.

\section{3-TNBC HAS A HIGHER RATE OF RESIDUAL DISEASE AFTER LUMPECTOMY}

TNBC is more common in younger women who may have smaller, more cosmetically driven surgeries as well as denser breasts that may harbor mammographically occult disease leading to an increased residual disease burden following lumpectomy (Tyler et al., 1995; Marin-Gutzke and Sanchez-Olaso, 2010; Pinsky and Helvie, 2010). This may result in increased residual disease after lumpectomy and subsequent increased risk of LR. To address this hypothesis we performed a retrospective pathologic chart review that revealed that TNBC are at increased risk of having residual disease after lumpectomy. We obtained pathologic data from nearly 400 women who had a lumpectomy for invasive cancer followed by a re-excision (or 
mastectomy) within a 3-month period. Descriptive statistics of the cohort by molecular phenotype are shown in Table 3. On univariate analysis, age, tumor size, margin status, lymph node involvement, LVI, grade, multifocality, and molecular phenotype were all correlated with residual invasive disease (Table 4). On forward selection, step-wise multivariate analysis, only lymph node involvement, tumor size, and TN phenotype maintained statistical significance as independent predictors of residual invasive disease (Table 5).

\begin{tabular}{llll}
\hline $\begin{array}{l}\text { Table } 3 \text { | Descriptive statistics by molecular phenotype in the } \\
\text { retrospective pathologic chart review examining rate of residual } \\
\text { disease after lumpectomy. }\end{array}$ & & \\
\hline $\boldsymbol{n = 3 4 5}$ & $\begin{array}{l}\text { Luminal A and } \\
\text { B, } \boldsymbol{n = 2 8 6}\end{array}$ & $\begin{array}{l}\text { HER2-enriched, } \\
\boldsymbol{n = 1 6}\end{array}$ & $\begin{array}{l}\text { Triple } \\
\text { negative, }\end{array}$ \\
& & & $\boldsymbol{n}=\mathbf{4 3}$ \\
& 29 & 24 & 50 \\
\hline Residual disease (\%) & 59 & 57 & 50 \\
Age (y) & 1.5 & 1.2 & 1.6 \\
Tumor size (cm) & 30 & 31 & 32 \\
Multifocal (\%) & 32 & 27 & 40 \\
Node+(\%) & 33 & 32 & 28 \\
Margin+(\%) & 35 & 63 & 43 \\
EIC (\%) & 20 & 19 & 30 \\
LVI (\%) & 19 & 86 & 74 \\
High grade (\%) & 1 & 50 & 56 \\
High mitotic count (\%) & &
\end{tabular}

EIC, extensive intraductal component; LVI, lymphovascular invasion.

Table 4 | Univariate analysis relating risk factors associated with local recurrence risk with incidence of residual disease found in the re-excision specimen after lumpectomy.

\begin{tabular}{lllc}
\hline Variable & $\boldsymbol{n}$ & OR $(\mathbf{9 5} \% \mathbf{C l})$ & $\boldsymbol{p}$-Value \\
\hline Triple negative & 343 & $2.48(1.30,4.74)$ & 0.006 \\
Node + & 345 & $3.69(2.29,5.97)$ & $<0.0001$ \\
Multifocal & 367 & $1.78(1.12,2.85)$ & 0.02 \\
LVI+ & 367 & $2.56(1.53,4.28)$ & 0.0004 \\
Age $>45$ & 365 & $0.45(0.27,0.76)$ & 0.003 \\
Margins + & 363 & $1.76(1.12,2.76)$ & 0.01 \\
High grade & 346 & $2.14(1.32,3.47)$ & 0.002 \\
Tumor size & 366 & $<1.0 \mathrm{~cm}:$ reference & 0.0005 \\
& & $1.1-2 \mathrm{~cm}: 3.00(1.62,5.54)$ & $<0.0001$ \\
\end{tabular}

OR, odds ratio; LVI, lymphovascular invasion.

Table 5 | Multivariate analysis identifying independent risk factors for having residual disease found in the re-excision specimen after lumpectomy.

\begin{tabular}{lcc}
\hline Variable & OR $(\mathbf{9 5} \% \mathbf{C l})$ & $\boldsymbol{p}$-Value \\
\hline Nodal + & $3.06(1.77-5.30)$ & $<0.0001$ \\
TN status & $3.28(1.56-6.89)$ & 0.002 \\
Tumor size & $3.49(1.65-7.38)$ & 0.001 \\
\hline
\end{tabular}

OR, odds ratio; $T N$, triple negative.
The finding that TNBCs harbor more microscopic residual disease after lumpectomy suggests that these patients may benefit from dose escalation. The European Organization for Research and Treatment for Cancer (EORTC) 22881 trial examined the benefit of tumor bed boost (defined as additional radiation dose delivered to the tumor bed) to BCT in over 5,000 women (Bartelink et al., 2007). With over 10 years of follow-up, LR rates were $10.2 \%$ in the whole breast irradiation only arm and $6.2 \%$ in the boost arm $(p<0.0001)$ with a hazards ratio of 0.59 . The statistically significant local control benefit of boost was maintained for all age groups although most dramatic in the younger age groups. Currently, the National Comprehensive Cancer Network (NCCN) recommends tumor bed boost for women at higher risk for recurrence including women $<50$ years old, positive axillary lymph nodes, close surgical margins, or LVI ${ }^{1}$. Subgroup analysis of the EORTC 22881 boost vs. no boost trial showed that the largest proportional benefit of boost was in the high grade tumors and in the patients under the age of 50 (Jones et al., 2009). Women with TNBC are more frequently young patients and overwhelmingly have high grade tumors. Therefore, the subgroups that were found to have a more robust local control benefit from tumor bed boost likely contained a higher proportion of TN disease.

Radiation oncologists should strongly consider tumor bed boost for TNBC to mitigate the increased risk of LR. Although TNBC is associated with an increased risk of residual disease after lumpectomy, the risk of recurrence is still acceptably low to support breast conservation. Therefore, TNBC is not an indication for mastectomy.

\section{APBI AND MOLECULAR PHENOTYPE}

Accelerated partial breast irradiation is an alternative technique to standard whole breast irradiation. With APBI the radiation target is limited to the tissues surrounding the tumor bed and the dose is hypofractionated (fewer total number of fractions generally with larger dose per fraction) and delivered over an accelerated time course (generally 1 week). There is evolving data APBI studies demonstrating a correlation between local failure and molecular phenotype. NSABP B-39/RTOG 0413, a randomized trial comparing whole breast irradiation to APBI, has been slow to enroll ER-negative patients ${ }^{2}$. This is likely due to conservative bias among oncologists regarding concern for a higher risk of LR in patients with ER-negative disease. Currently, the American Society for Radiation Oncology (ASTRO) consensus guidelines designated ER-negative status a "cautionary" criteria for APBI and strongly encourages the enrollment of ER-negative patients in the trial (Smith et al., 2009).

The literature on APBI and breast cancer subtype is evolving and mixed. There are at least two reports that do not show any difference in LR after APBI by subtype. The first is a by Wilder et al. (2010) and is limited by short follow-up of only 22 months. They analyzed 209 women treated with APBI and had excellent local control rates of $99-100 \%$ for all subtypes, indicating careful patient selection and short follow-up. However, a second report by Wilkinson et al. (2011) showed similar results with a mean of 4.1 years of followup. They reported no LR in 20 TNBC patients treated with APBI. In contrast, Pashtan et al. (2010) reported on a series of 99 Stage I patients treated with APBI and found that patients with TN tumors

${ }^{1}$ http://www.nccn.com/patient-guidelines.html\#breast ${ }^{2}$ http://www.rtog.org/ClinicalTrials/ProtocolTable/StudyDetails.aspx?study=0413 
had a significantly higher rate of LR compared to other subtypes. With a median follow-up of 47.5 months, they had an overall 5 year actuarial LR rate of $6 \%$. Three of the six LR occurred in TN patients, yielding a LR of $32.5 \%$ among TN patients compared to only $3 \%$ among the other subtypes $(p=0.0001)$. Interestingly, only one out of the six LR occurred in the same quadrant, and the other five were deemed elsewhere LR. In a report out of the University of Wisconsin, of 136 "cautionary" status patients treated with APBI, 11 were ER-negative. After a median follow-up of 60 months, they reported a total of five IBTR. Two of the five IBTR were in ER-negative patients, yielding a failure rate of $18 \%$ in ER-negative patients compared to only $2.4 \%$ among the ER-positive patients (McHaffie et al., 2010). Additionally, ER-negative status was the only factor that correlated with IBTR after APBI in the 1,225 cases from the Mammosite ${ }^{\circledR}$ Registry (Beitsch et al., 2010).

\section{LUMINAL SUBTYPE HETEROGENEITY AND 21-GENE RECURRENCE SCORE ASSAY}

In addition to the identification of molecular phenotypes in breast cancer, gene recurrence score assays are emerging and may be useful in predicting the risk of LR. One example is a 21-gene recurrence score assay (Oncotype DX, Genomic Health, Redwood City, CA, USA) that was developed through the prospective selection of genes that were thought to correlate with distant recurrence risk in node-negative, ER(+) breast cancer (Paik et al., 2004). Initially it was designed to be a prognostic tool to quantify the risk of distant recurrence. It was later found to also be predictive of the benefit of adjuvant chemotherapy as well as the risk of LR (Paik et al., 2006). Mamounas et al. (2010) reported a statistically significant association between recurrence score (RS) and LRR in the NSABP B-14 and B-20 datasets of 895 patients. They found that 10 -year LRR was $4.3 \%$ for patients with low RS (<18), 7.2\% for intermediate risk RS (Haffty et al., 2006; Dent et al., 2007; Cheang et al., 2008; Crabb et al., 2008; Kyndi et al., 2008; Nguyen et al., 2008; Foulkes et al., 2009; Freedman et al., 2009; Millar et al., 2009; Albert et al., 2010; Billar et al., 2010; Voduc et al., 2010), and $15.8 \%$ for high RS ( $>30 ; p<0.001)$. Thus, incorporating RS into local management decision making for luminal/ER(+) subtypes may be useful and can potentially help distinguish which patients with luminal breast cancer are at increased risk of LR.

\section{DCIS AND MOLECULAR PHENOTYPE}

The prognostic significance of molecular markers in ductal carcinoma in situ (DCIS) has been an area of ongoing research. HER2 expression has been found to correlate with aggressive histologic features (Cornfield et al., 2004; Stackievicz et al., 2010) such as high nuclear grade and comedo necrosis. However, unlike invasive breast cancer, the prognostic significance of HER2 expression in DCIS has not been demonstrated. Stackievicz et al. (2010) reported

\section{REFERENCES}

Albert, J. M., Gonzalez-Angulo, A. M., Guray, M., Sahin, A., Strom, E. A., Tereffe, W., Woodward, W. A., Tucker, S. L., Hunt, K. K., Hortobagyi, G. N., and Buchholz, T. A. (2010). Estrogen/ progesterone receptor negativity and HER2 positivity predict locoregional recurrence in patients with $\mathrm{Ta} 1, \mathrm{Bn} 0$ breast cancer. Int. J. Radiat. Oncol. Biol. Phys. 77, 1296-1302.

Bartelink, H., Horiot, J. C., Poortmans, P. M., Struikmans, H., Van den Bogaert, W., Fourquet, A., Jager, J. J., Hoogenraad, W. J., Oei, S. B., Wárlám-Rodenhuis, C. C., Pierart, M., and Collette, L. (2007). Impact of higher radiation dose on local

no correlation between HER2 expression in 84 DCIS patients and disease recurrence. However, this study was limited by small numbers and a possibly over-inclusive definition of HER2-positivity (IHC membrane staining of $>10 \%$ of cells and did not perform FISH; Stackievicz et al., 2010). Although the prognostic significance of HER2 expression in DCIS remains unclear, the receptor is still a viable marker that can be targeted with trastuzumab and may potentially impact the management of the disease. Kuerer et al. (2011) reported on a phase II trial conducted at MDACC on patients with HER2-positive DCIS treated with a single dose of preoperative trastuzumab. They defined HER2-positivity as IHC $3+$ or by FISH amplification. They did not find any significant, clinically overt, histologic, antiproliferative, or apoptotic changes. However, trastuzumab did appear to mount antibody-dependent cell mediated cytotoxicity and possible induction of T-cell dependent humoral immunity (Kuerer et al., 2011). The role of trastuzumab is also being investigated in the postoperative setting for treatment of DCIS. NSABP B-43 is a phase III trial currently enrolling DCIS patients and randomizing them to radiation with out without concurrent trastuzumab after lumpectomy ${ }^{3}$.

The prognostic significance of molecular phenotype in DCIS has also been studied. Zhou et al. (2010) reported results of a cohort study of 392 women with primary DCIS who had complete IHC data available for the ER, PR, HER2, EGFR, and CK 5/6 markers (Zhou et al., 2010). Molecular phenotype was defined similar to the definitions used in invasive cancer. The $8.2 \%$ were basal-like DCIS (negative for ER, HER 2 and positive for EGFR and/or CK 5/6), 89.5\% were luminal or HER2+, and $2.3 \%$ were unclassified (negative for all markers). On univariate and multivariate analysis, with over 8 years of median follow-up, basal-like DCIS showed a higher risk of LR and invasive recurrence compared to non-basal-like DCIS but this result was not statistically significant ( $p=0.08$ and $p=0.15$, respectively). Further studies evaluating molecular phenotype and DCIS are warranted.

\section{CONCLUSION}

Molecular profiling is an area of rapid growth in breast cancer research and will continue to evolve over time. Although the correlation between molecular profiles and LRR are compelling, validation with larger independent datasets and prospective randomized trials are still necessary before we can make any definitive conclusions or make any major deviations from our current clinical practice. The studies reviewed in this paper should be considered hypothesis generating. Factoring molecular phenotype and recurrence scores into adjuvant treatment decision making, along with the traditional histopathologic and clinical risk factors, may be appropriate in select situations.

${ }^{3}$ http://www.nsabp.pitt.edu/B43_Information.asp

control and survival in breastconserving therapy of early breast cancer: 10-year results of the randomized boost versus no boost EORTC 22881-10882 trial. J. Clin. Oncol. 25, 1-10.

Bauer, K. R., Brown, M., Cress, R. D., Parise, C. A., and Caggiano, V. (2007). Descriptive analysis of estrogen receptor (ER)-negative, progesterone receptor $(\mathrm{PR})$-negative and HER2negative invasive breast cancer, the so-called triple-negative phenotype: a population-based study from the Califonia cancer registry. Cancer 109 , 1721-1728.

Beitsch, P., Vicini, F., Keisch, M., Haffty, B., Shaitelman, S., and Lyden, M. (2010). 
Five-year outcome of patients classified in the "unsuitable" category using the American society of therapeutic radiology and oncology consensus panel guidelines for the application of accelerated partial breast irradiation: an analysis of patients treated on the American society of breast surgeons MammoSite registry trial. Ann. Surg. Oncol. 17, S219-S225.

Billar, J. A., Dueck, A. C., Stucky, C. C. H., Gray, R. J., Wasif, N., Northfelt, D. W., McCullough, A. E., and Pockaj, B. A. (2010). Triple-negative breast cancers: unique clinical presentations and outcomes. Ann. Surg. Oncol. 17, S384-S390.

Carey, L. A., Perou, C. M., Livasy, C. A., Dressler, L. G., Cowan, D., Conway, K., Karaca, G., Troester, M. A., Tse, C. K., Edmiston, S., Deming, S. L., Geradts, J., Cheang, M. C., Nielsen, T. O., Moorman, P. G., Earp, H. S., and Millikan, R. C. (2006). Race, breast cancer subtypes, and survival in the Carolina breast cancer study. J. Am. Med. Assoc. 295, 2492-2502.

Cheang, M. C., Voduc, D., Bajdik, C., Leung, S., McKinney, S., Chia, S. K., Perou, C.M., and Nielsen, T.O. (2008). Basal-like breast cancer defined by five biomarkers has superior prognostic value than triple-negative phenotype. Clin. Cancer Res. 14, 1368-1376.

Clarke, D., and Martinez, A. (1992). Identification of patients who are at high risk for locoregional breast cancer recurrence after conservative surgery and radiotherapy: a review article for surgeons, pathologists, and radiation and medical oncologists. J. Clin. Oncol. 10, 474-483.

Cornfield, D. B., Palazzo, J. P., Schwartz, G. F., Goonewardene, S. A., Kovatich, A. J., Chervoneva, I., Hyslop, T., and Schwarting, R. (2004). The prognostic significance of multiple morphologic features and biologic markers in ductal carcinoma in situ of the breast. Cancer 100, 2317-2327.

Crabb, S. J., Cheang, M. C., Leung, S., Immonen, T., Nielsen, T. O., Huntsman, D. D., Bajdik, C. D., and Chia, S. K. (2008). Basal breast cancer molecular subtype predicts for lower incidence of axillary lymph node metastases in primary breast cancer. Clin. Breast Cancer 8, 249-256.

Dent, R., Trudeau, M., Prichard, K. I.,Hanna, W. M., Kahn, H. K., Sawka, C. A., Lickley, L. A., Rawlinson, E., Sun, P., and Narod, S. A. (2007). Triple-negative breast cancer: clinical features and patterns of recurrence. Clin. Cancer Res. 13, 4429-4434.

Fan, C., Oh, D. S., Wessels, L., Weigelt, B., Nuyten, D. S., Nobel, A. B., van't Veer, L. J., and Perou, C. M. (2006).
Concordance among gene-expression-based predictors for breast cancer. N. Engl. J. Med. 355, 560-569.

Fisher, B., Redmond, C., Brown, A., Wickerham, D. L., Wolmark, N., Allegra, J., Escher, G., Lippman, M., Savlov, E., and Wittliff, J. (1983). Influence of tumor estrogen and progesterone receptor levels on the response to tamoxifen and chemotherapy in primary breast cancer. $J$. Clin. Oncol. 1, 227-241.

Foulkes, W. D., Grainge, M. J., Rakha, E. A., Green, A. R., and Ellis, I. O. (2009). Tumor size is an unreliable predictor of prognosis in basal-like breast cancers and does not correlate closely with lymph node status. Breast Cancer Res. Treat. 117, 199-204.

Freedman, G. M., Anderson, P. R., Li, T., and Nicolaou, N. (2009). Locoregional recurrence of triplenegative breast cancer after breastconserving surgery and radiation. Cancer 115, 946-951. [Author correspondence published in Cancer January 15, 2009 Vol. 116, 538-539 (Comment by Fabio Puglisi with response by Gary M. Freedman)].

Haffty, B. G., Yang, Q., Reiss, M., Kearney, T., Higgins, S. A., Weidhaas, J., Harris, L., Hait, W., and Toppmeyer, D. (2006). Locoregional relapse and distant metastasis in conservatively managed triple negative early-stage breast cancer. J. Clin. Oncol. 24, 5652-5657.

IBTR! (2011). Version 2.0 Breast Cancer Model. Available at: http://160.109.101.132/ibtr/ [accessed March 14, 2011].

Jones, H. A., Antonini, N., Hart, A. A. M., Peterse, J. L., Horiot, J. C, Collin, F., Poortmans, P. M., Oei, S. B., Collette, L., Struikmans, H., Van den Bogaert, W. F., Fourquet, A., Jager, J. J., Schinagl, D. A., Wárlám-Rodenhuis, C. C., and Bartelink, H. (2009). Impact of pathologic characteristic on local relapse after breast-conserving therapy: a subgroup analysis of the EORTC boost vs no boost trial. J. Clin. Oncol. 27, 4939-4947.

Kuerer, H. M., Buzdar, A. U., Mittendorf, E. A., Esteva, F. J., Lucci, A., Vence, L. M., Radvanyi, L., Meric-Bernstam, F., Hunt, K. K., and Symmans, W. F. (2011). Biologic and immunologic effects of preoperative trastuzumab for ductal carcinoma in situ of the breast. Cancer 117, 39-47.

Kyndi, M., Overgaard, M., Nielson, M., Sørensen, F. B., Knudsen, H., and Overgaard, J. (2009). High local recurrence risk is not associated with large survival reduction after postmastectomy radiotherapy in high-risk breast cancer: a subgroup analysis of DBCG 82 b\&c. Radiother. Oncol. 90, 74-79.
Kyndi, M., Sorensen, F. B., Knudsen, H., Overgaard, M., Nielsen, H. M., and Overgaard, J. (2008). Estrogen receptor, progesterone receptor, HER-2, and response to postmastectomy radiotherapy in high-risk breast cancer: the Danish Breast Cancer Cooperative Group. J. Clin. Oncol. 26, 1419-1426. Mamounas, E. P., Tang, G., Fisher, B., Paik, S., Shak, S., Costantino, J. P., Watson, D., Geyer, C. E. Jr., Wickerham, D. L., and Wolmark, N. (2010). Association between the 21-gene recurrence score assay and risk of locoregional recurrence in node-negative, estrogen receptor-positive breast cancer: results from NSABP B-14 and NSABP B-20. J. Clin. Oncol. 28, 1677-1683.

Marin-Gutzke, M., and Sanchez-Olaso, A. (2010). Reconstructive surgery in young women with breast cancer. Breast Cancer Res. Treat 123(Suppl. 1), 67-74.

McHaffie, D. R., Patel, R. R., Adkison, J. B. Das, R. K., Geye, H. M., and Cannon, G. M. (2010). Outcomes after accelerated partial breast irradiation in patients with ASTRO consensus statement cautionary features. Int. J. Radiat. Oncol.Biol.Phys. [Epub ahead of print].

Millar, E. K., Graham, P.H., O’Toole, S.A., McNeil, C.M., Browne, L., Morey, A. L., Eggleton, S., Beretov, J., Theocharous, C., Capp, A., Nasser, E., Kearsley, J. H., Delaney, G., Papadatos, G., Fox, C., and Sutherland, R. L. (2009). Prediction of local recurrence, distant metastases, and death after breast-conserving therapy in early-stage invasive breast cancer using a five-biomarker panel. $J$. Clin. Oncol. 27, 4701-4708.

Millikan, R. C., Newman, B., Tse, C. K. Moorman, P. G., Conway, K., Dressler, L. G., Smith, L. V., Labbok, M. H., Geradts, J., Bensen, J. T., Jackson, S., Nyante, S., Livasy, C., Carey, L., Earp, H. S., and Perou, C. M. (2008). Epidemiology of basal-like breast cancer. Breast Cancer Res. Treat. 109, 123-139.

Nguyen, P. L., Taghian, A. G., Katz, M. K., Niemierko, A., Raad, R. F.A., Boon, W. L., Bellon, J. R., Wong, J. S., Smith, B. L., and Harris, J. R. (2008). Breast cancer subtype approximated by estrogen receptor, progesterone receptor, and HER-2 is associated with local and distant recurrence after breast-conserving therapy. J. Clin. Oncol. 26, 2373-2378.

Nielsen, T. O., Hsu, F. D., Jensen, K., Cheang, M., Karaca, G., Hu, Z., Hernandez-Boussard, T., Livasy, C., Cowan, D., Dressler, L., Akslen, L. A., Ragaz, J., Gown, A. M., Gilks, C. B., van de Rijn, M., and Perou, C. M. (2004). Immunohistochemical and clinical characterization of the basal-like subtype of invasive breast carcinoma. Clin. Cancer Res. 10 5367-5374.

Overgaard, M., Hansen, P. S., Overgaard, J., Rose, C., Andersson, M., Bach, F., Kjaer, M., Gadeberg, C. C., Mouridsen, H. T., Jensen, M. B., and Zedeler, K. (1997). Postoperative radiotherapy in high-risk premenopausal women with breast cancer who receive adjuvant chemotherapy. N. Engl. J. Med. 337, 949-955.

Overgaard, M., Hensen, M. B., Overgaard, J., Hansen, P. S., Rose, C., Andersson, M., Kamby, C., Kjaer, M., Gadeberg, C. C., Rasmussen, B. B., Blichert-Toft, M., and Mouridsen, H. T. (1999). Postoperative radiotherapy in highrisk postmenopausal breast-cancer patients given adjuvant tamoxifen: Danish Breast Cancer Cooperative Group DBCG 82c randomised trial. Lancet 353, 1641-1648.

Paik, S., Shak, S., Tang, G., Kim, C., Baker, J., Cronin, M., Baehner, F. L., Walker, M. G., Watson, D., Park, T., Hiller, W., Fisher, E. R., Wickerham, D. L., Bryant, J., and Wolmark, N. (2004). A multigene assay to predict recurrence of tamoxifen-treated, node-negative breast cancer. N. Engl. J. Med. 351, 2817-2826.

Paik, S., Tang, G., Shak, S., Kim, C., Baker, J., Kim, W., Cronin, M., Baehner, F. L., Watson, D., Bryant, J., Costantino, J. P., Geyer, C. E. Jr., Wickerham, D. L., and Wolmark, N. (2006). Gene expression and benefit of chemotherapy in women with node-negative, estrogen receptor-positive breast cancer. J. Clin. Oncol. 24, 3726-3734.

Parker, J., Mullins, M., Cheang, M. C., Leung, S., Voduc, D., Vickery, T., Davies, S., Fauron, C., He, X., Hu, Z., Quackenbush, J. F., Stijleman, I. J., Palazzo, J., Marron, J. S., Nobel, A. B., Mardis, E., Nielsen, T. O., Ellis, M. J., Perou, C. M., and Bernard, P.S. (2009). A supervised risk predictor of breast cancer based on intrinsic subtypes. J. Clin. Oncol. 27, 1160-1167.

Pashtan, I. M., Ancukiewicz, M., Wo, J. Y., Hirsch, A. E., Smith, B. L., Powell, S. N., Recht, A., and Taghian, G. (2010). Triple-negative breast cancer is associated with higher risk of local recurrence after $3 \mathrm{D}$-conformal external beam accelerated partial breast irradiation (3D-APBI) Abstract presented at 52nd Annual ASTRO Meeting, San Diego, CA. [Int. J. Radiat. Oncol. Biol. Phys. 78, S53].

Perou, C. M., Sorlie, T., Eisen, M. B., van de Rijn, M., Jeffrey, S. S., Rees, C. A., Pollack, J. R., Ross, D. T., Johnsen, H., Akslen, L. A., Fluge, O., Pergamenschikov, A., Williams, C., 
Zhu, S. X., Lønning, P. E., BørresenDale, A. L., Brown, P. O., and Botstein, D. (2000). Molecular portraits of human breast tumours. Nature 406, 747-752.

Piccart-Gebhart, M. J., Procter, M., Leyland-Jones, B., Goldhirsch, A., Untch, M., Smith, I., Gianni, L., Baselga, J., Bell, R., Jackisch, C., Cameron, D., Dowsett, M., Barrios, C. H., Steger, G., Huang, C. S., Andersson, M., Inbar, M., Lichinitser, M., Láng, I., Nitz, U., Iwata, H., Thomssen, C., Lohrisch, C., Suter, T. M., Rüschoff, J., Suto, T., Greatorex, V., Ward, C., Straehle, C., McFadden, E., Dolci, M. S,, Gelber, R. D., and Herceptin Adjuvant (HERA) Trial Study Team. (2005). Trastuzumab after adjuvant chemotherapy in HER2-positive breast cancer. N. Engl. J. Med. 353, 1659-1672.

Pinsky, R. W., and Helvie, M. A. (2010). Mammographic breast density: effect on imaging and breast cancer risk. J. Natl. Compr. Canc. Netw. 10, 1157-1164.

Romond, E. H., Perez, E. A., Bryant, J., Suman, V. J., Geyer, C. E. Jr., Davidson, N. E., Tan-Chiu, E., Martino, S., Paik, S., Kaufman, P. A., Swain, S. M. Pisansky, T. M., Fehrenbacher, L., Kutteh, L. A., Vogel, V. G., Visscher, D. W., Yothers, G., Jenkins, R. B., Brown, A. M., Dakhil, S. R., Mamounas, E. P., Lingle, W. L., Klein, P. M., Ingle, J. N., and Wolmark, N. (2005). Trastuzumab plus adjuvant chemotherapy for operable HER2-positive breast cancer. $N$. Engl. J. Med. 353, 1673-1684.

Sachdev, J. C., Ahmed, S., Mirza, M. M., Farooq, A., Kronish, L., and Jahanzeb, M. (2010). Does race affect outcomes in triple negative breast cancer? Breast Cancer (Auckl.) 4, 23-33.
Slamon, D. J., Clark, G. M., Wong, S. G., Levin, W. J., Ullrich, A., and McGuire, W. L. (1987). Human breast cancer: correlation of relapse and survival with amplification of the HER-2/neu oncogene. Science 235, 177-182.

Smith, B. D., Arthur, D. W., Buccholz, T. A., Haffty, B. G., Hahn, C. A., Hardenbergh, P. H., Julian, T. B., Marks, L. B., Todor, D. A., Vicini, F. A., Whelan, T. J., White, J., Wo, J. Y., and Harris, J. R. (2009). Accelerated partial breast irradiation consensus statement from the American Society for Radiation Oncology (ASTRO). Int. J. Radiat. Oncol. Biol. Phys. 74, 987-1001.

Sorlie, T. (2004). Molecular portraits of breast cancer: tumour subtypes as distinct disease entities. Eur. J. Cancer 40, 2667-2675.

Sorlie, T., Perou, C. M., Tibshirani, R., Aas, T., Geisler, S., Johnsen, H., Hastie, T., Eisen, M. B., van de Rijn, M., Jeffrey, S. S., Thorsen, T., Quist, H., Matese, J. C., Brown, P. O., Botstein, D., Eystein Lønning, P., and Børresen-Dale, A. L. (2001). Gene expression patterns of breast carcinomas distinguish tumor subclasses with clinical implications. Proc. Natl. Acad. Sci. U.S.A. 98, 10869-10874.

Sorlie, T., Tibshirani, R., Parker, J., Hastie, T., Marron, J. S., Nobel, A., Deng, S., Johnsen, H., Pesich, R., Geisler, S., Demeter, J., Perou, C. M., Lønning, P. E., Brown, P. O., Børresen-Dale, A. L., and Botstein, D (2003). Repeated observation of breast tumor subtypes in independent gene expression data sets. Proc. Natl. Acad. Sci. U.S.A. 100, 8418-8423.

Sotiriou, C., Neo, S. Y., McShane, L. M. Korn, E. L., Long, P. M., Jazaeri, A.,
Martiat, P., Fox, S. B., Harris, A. L. and Liu, E. T. (2003). Breast cancer classification and prognosis based on gene expression profiles from a population-based study. Proc. Natl. Acad. Sci. U.S.A. 100, 10393-10398.

Stackievicz, R., Paran, H., Bernheim, J. Shapira, M., Weisenberg, N., Kaufman, T., Klein, E., and Gutman, M (2010) Prognostic significance of HER-2.neu expression in patients with ductal carcinoma in situ. IMAJ 12, 290-295.

Tyler, M. E., Perez, C. A., Halverson, K. J. Kuske, R. R., Philpott, G. W., Garcia, D. M., Mortimer, J. E., Myerson, R. J., Radford, D., and Rush, C. (1995) Factors influencing cosmetic results after conservation therapy for breast cancer. Int. J. Radiat. Oncol. Biol. Phys. 31, 753-764.

van't Veer, L. J., Dai, H., van de Vijver, M. J., He, Y. D., Hart, A. A. M., Mao, M. Peterse, H.L., van der Kooy, K., Marton, M. J., Witteveen, A. T., Schreiber, G. J., Kerkhoven, R. M., Roberts, C., Linsley, P. S., Bernards, R., and Friend, S. H (2002). Gene-expression profiling predicts clinical outcome of breast cancer. Nature 415, 530-536.

Voduc, K. D., Cheang, M. C., Tyldesley, S., Gelmon, K., Nielsen, T. O., and Kennecke, H. (2010). Breast cancer subtypes and the risk of local and regional relapse. J. Clin. Oncol. 28, 1684-1691.

Wilder, R. B., Curcio, L. D., Khanijou, R. K., Eisner, M. E., Kakkis, J. L., Chittenden, L., Agustin, J., Lizarde, J., Mesa, A. V., Macedo, J. C., Ravera, J. and Tokita, K. M. (2010). Results with accelerated partial breast irradiation in terms of estrogen receptor, progesterone receptor, and human growth factor receptor 2 status. Int. J. Radiat. Oncol. Biol. Phys. 78, 799-803.
Wilkinson, J. B., Reed, R. E., Wallace, M. F., Chen, P. Y., Mitchell, C. K., Shaitelman, S. F., Marvin, K. S., Grills, I. S., Margolis, J. M., and Vicini, F. A. (2011). Outcomes of breast cancer patients with triple negative receptor status treated with accelerated partial breast irradiation. Int. J. Radiat Oncol. Biol. Phys. [Epub ahead of print].

Zhou, W., Jirstrom, K, Johansson, C. Amini, R. M., Blomqvist, C., Agbaje, O., and Wärnberg, F. (2010). Longterm survival of women with basallike ductal carcinoma in situ of the breast: a population-based cohort study. BMC Cancer 10, 653-660. doi: 10.1186/1471-2407-10-653

Conflict of Interest Statement: The authors declare that the research was conducted in the absence of any commercial or financial relationships that could be construed as a potential conflict of interest.

Received: 22 March 2011; paper pending published: 14 April 2011; accepted: 12 June 2011; published online: 28 June 2011.

Citation: Sioshansi S, Huber KE and Wazer DE (2011) The implications of breast cancer molecular phenotype for radiation oncology. Front. Oncol. 1:12. doi: 10.3389/ fonc.2011.00012

This article was submitted to Frontiers in Radiation Oncology, a specialty of Frontiers in Oncology.

Copyright (c) 2011 Sioshansi, Huber and Wazer. This is an open-access article subject to a non-exclusive license between the authors and Frontiers Media SA, which permits use, distribution and reproduction in other forums, provided the original authors and source are credited and other Frontier conditions are complied with. 